\title{
Implementation of a Cost-Accounting Model in a Biobank: Practical Implications
}

\author{
Maria Beatriz Gonzalez-Sanchez ${ }^{\mathrm{a}}$ Ernesto Lopez-Valeiras ${ }^{\mathrm{a}}$ \\ Andres C. García-Montero ${ }^{\text {b }}$ \\ ${ }^{a}$ Faculty of Business Administration and Tourism, University of Vigo, Ourense, and b National DNA Bank Carlos III, \\ Cancer Research Center (IBMCC), University of Salamanca, Salamanca, Spain
}

\section{Key Words}

Costs · Biobanks · Financial sustainability

\begin{abstract}
Given the state of global economy, cost measurement and control have become increasingly relevant over the past years. The scarcity of resources and the need to use these resources more efficiently is making cost information essential in management, even in non-profit public institutions. Biobanks are no exception. However, no empirical experiences on the implementation of cost accounting in biobanks have been published to date. The aim of this paper is to present a step-by-step implementation of a cost-accounting tool for the main production and distribution activities of a real/active biobank, including a comprehensive explanation on how to perform the calculations carried out in this model. Two mathematical models for the analysis of (1) production costs and (2) request costs (order management and sample distribution) have stemmed from the analysis of the results of this implementation, and different theoretical scenarios have been prepared. Global analysis and discussion provides valuable information for internal biobank management and even for strategic decisions at the research and development governmental policies level.

(c) 2015 S. Karger AG, Basel
\end{abstract}

\section{Introduction}

Cost-accounting design and implementation involves a non-trivial challenge for biobank managers. Biobanking entails complex and high resource-consuming production processes. Moreover, this activity is subject to an unpredictable demand and long-cycle storage times. Cost models previously developed for health care institutions (e.g. $[1,2])$ are not suitable for biobanks due to the intrinsic characteristics of the biobank production process, its 'market' conditions and its external environment. While some specific biobanking management issues have been previously examined in the literature (e.g. $[3,4])$, to the best of our knowledge, there is only one study (by Gonzalez-Sanchez et al. [5]) that proposes a theoretical cost model specifically designed to be implemented in biobanks. However, it lacks the actual description and analysis of implementation in a biobank. Drawing on Gonzalez-Sanchez et al. [5] and following the recommendations of International Public Sector Accounting Standard (IPSAS) 12 [6], the aim of this paper is to present a step-by-

All authors have contributed equally to this work and all should be considered as first/last authors.

\section{KARGER}

E-Mail karger@karger.com www.karger.com/pat (c) 2015 S. Karger AG, Base

$1015-2008 / 15 / 0816-0286 \$ 39.50 / 0$
Dr. Maria Beatriz Gonzalez-Sanchez Accounting and Finance Department Faculty of Business Administration and Tourism, University of Vigo Edificio Jurídico-Empresarial, s/n, ES-32004 Ourense (Spain)

E-Mail bgonzale@uvigo.es 
step implementation procedure of a cost-accounting tool for the main production activity in a real/active biobank. The analysis and discussion of the results will help to uncover data concerning the real financial sustainability of these kinds of organizations and the extent to which financial investments in biobanks actually contribute to modern biomedical research $[4,7]$. Additionally, implementing a cost-accounting system, even in a non-profit biobank, will provide biobank managers with valuable information that could represent a key management tool for taking decisions concerning economic and technical issues associated with biobanks and biobank activities; thus, it could indirectly improve the financial sustainability of the organization [8].

The Spanish National DNA Bank Carlos III [Banco Nacional de $\mathrm{ADN}$ (BNADN)] was the institution chosen to represent the implementation of the model, given (1) their long experience in biobanking activities, (2) the large numbers of collections of biological samples stored in it, (3) the great number of products they have distributed over the course of the past 9 years $(>80,000$ sample aliquots for more than 100 different research projects), (4) their activities (certified under the ISO 9001 quality management system since 2006) in the extraction and storage of nucleic acids and the management of data associated with samples, as a service for research groups working in biomedical areas, (5) their collaboration with a national biobanking hub (Spanish Biobanking Network) where analysing the real cost of biobanking activities is a specific goal in guaranteeing the long-term sustainability of biobanking, and (6) their interest, with a clear involvement of both managers and employees, in applying this management tool.

By implementing cost accounting, the BNADN obtained information about (1) the cost of processing each case (the set of biomaterials collected from a donor at a specific time point), (2) the annual inventory maintenance cost, (3) the unitary cost of every type of sample stored (each different biological product obtained from a subject), (4) the cost of selecting and preparing sample aliquots to be shipped for an order, and (5) the cost of order management and shipping. This paper presents the operations for the year 2011 from an ex-post perspective.

\section{Materials and Methods}

\section{Biobank and Biobank Activities Evaluated}

The BNADN is a scientific and technological platform, promoted and supported by the Spanish Government through the National Institute of Health Carlos III (Instituto de Salud Carlos III). Its main goal is to sustain genetic/genomic research by providing high-quality biological human samples (mainly DNA) and associated data for individual researchers, research networks, consortia and institutions. Moreover, it ensures that these samples and data are rationally as well as efficiently, ethically, legally and scientifically used.

Robotic technologies for DNA extraction and liquid sample handling are available to carry out all of the above activities. In turn, equipment and technology for different sample storage formats at room temperature, $4,-80$ and $-196^{\circ} \mathrm{C}$ are also available. Finally, a proprietary Laboratory Information Management Software (LIMS) for sample and data management has been developed to handle all of the information and data obtained from the moment a biospecimen (or data) is collected from an individual at a collaborating centre to the moment their products (i.e. sample aliquots and associated data) are distributed and used by external research groups. The quality of the stored DNA samples is assessed through different technologies to control DNA purity, integrity and sample traceability (e.g. electrophoresis/pulse-field electrophoresis, spectrophotometry, fluorimetry, multiplex and long-extent polymerase chain reaction, and a short tandem repeats test).

In order to ensure clarity and avoid wordiness, the model presented in this paper is limited to the most commonly requested type of products [DNA, plasma and peripheral blood mononuclear cells (PBMC)] obtained from volunteer donors (healthy volunteers or patients). Provision of other types of samples such as microRNA, mRNA, cDNA, urine, lymphoblastoid cell lines and purified cell populations requires the development of a specific production design for each request and, consequently, specific cost evaluation.

\section{Implementation of the Cost Model}

Drawing on the theoretical cost model for biobanks proposed by Gonzalez-Sanchez et al. [5], the first two steps for the implementation of the cost analysis cycle at BNADN included determination of the stages of the biobank activities and the cost objects.

Determination of the Stages of the Biospecimen Production

Process in the Biobank (BNADN)

First of all, the activities of the biobank were split into six stages. To facilitate the later understanding of the cost analysis, each stage is briefly described below.

Collection of Cases. Peripheral blood (PB) from volunteer donors is collected in blood collection tubes at the collaborating centres of the BNADN and transported to the biobank facility together with the epidemiological and health questionnaire of each individual and a copy of their consent report. Once received at the biobank, both biospecimens and associated data are re-codified, barcode-labelled and registered in the database associated with the LIMS of BNADN.

Processing of Biospecimens. Tubes containing $\mathrm{PB}$ are processed separately to obtain DNA and plasma [ethylenediaminetetraacetic acid (EDTA) collection tubes] as well as PBMC [acid citrate dextrose (ACD) tubes]. After centrifugation to separate the plasma from the cell fraction of blood, DNA is extracted by the salting-out method using an automated nucleic acid extractor ( $\mathrm{Au}$ topure LS, Qiagen). Afterward, extracted DNA is quantified (NanoDrop 1000, Thermo Scientific) and normalized at $100 \mathrm{ng} /$ $\mu \mathrm{l}$ using a liquid-handler workstation (Genesis, TECAN). In parallel, plasma is submitted to an additional centrifugation step to 
discard platelets and other cell debris. Then, both normalized DNA and plasma are archived on 2D-coded microtubes (Micronic $^{\circledR}$ ) using a liquid-handler workstation. PBMC are obtained after Ficoll density centrifugation and preserved in RPMI culture medium with $10 \%$ dimethyl sulfoxide (DMSO) and $20 \%$ foetal bovine serum (FBS) on cryovials identified with bar-coded ultralow temperature-resistant labels. All processes are tracked and registered in the LIMS.

Storage of Sample Products. Once obtained and archived in the appropriate tubes, the DNA, plasma and PBMC sample aliquots are placed at ultralow temperatures for long-term storage, either at $-80^{\circ} \mathrm{C}$ (e.g. DNA and plasma) or below $-170^{\circ} \mathrm{C}$ (e.g. PBMC). At BNADN, the average quantity of stored products per case includes 120 DNA sample aliquots (taking $1.5 \mu \mathrm{g}$ of DNA as the usual aliquot unit sent per order), 8 sample aliquots of $450-500 \mu \mathrm{l}$ of plasma and 2 sample aliquots of 10-20 × 10 6 PBC.

Order Management. A sample request can be sent by any researcher who prepares a scientific project approved by the BNADN external scientific and ethics committees. Once the order is received, the sample requirements requested by the researcher are analysed using the LIMS to check whether the sample collections stored at the biobank comply with the sample requests. If the order can be fulfilled with the inventory, then the scientific project is sent to the external scientific and ethical committees for approval. Only upon receiving the positive report from both of the external committees does the biobank directorate authorize the preparation and delivery of the samples and associated information.

Sample Selection and Preparation for Distribution. Stored sample products have to be selected according to their compliance with the requirements of researchers using the LIMS. When the information about the storage position of the available sample aliquots is obtained, samples have to be withdrawn from the freezers and their identity must be checked with a 2D tracker system or manual barcode reader (e.g. for PBMC cryovials stored in liquid nitrogen). Sample aliquots are prepared using the liquid-handler workstation and quality control is performed.

Distribution of Sample Aliquots. The selected sample aliquots that pass the quality test are then packed in standard biological biosafe (class B) containers to be sent to researchers in due course. All associated sample information is then sent to the researcher along with its pertinent biosafety sample management information. Samples are sent by priority courier service $(\leq 24 \mathrm{~h})$ to ensure they are transported and received under optimal conditions.

Determination of the Cost Objects at the Biobank (BNADN)

Next, starting from the general cost model for biobanks proposed by Gonzalez-Sanchez et al. [5] and taking into account the specific characteristics of the BNADN, five cost objects have been identified.

Case Processing. Under this cost object, all costs required to treat the tubes of $\mathrm{PB}$ received at the biobank for subsequent storage of DNA, plasma and PBMC products are included. It encompasses the stages of collecting and processing biospecimens, and associated information, from individual cases.

Inventory Maintenance per Year. This includes all the essential cost elements needed to preserve the products under optimal conditions until researchers request them for their research projects. This concept refers to all costs supported for storage, per year. The main cost elements are the depreciation of existing equipment, preventive maintenance of storage equipment, and consumption of electricity and nitrogen. It involves the storage of sample aliquots obtained after processing the biospecimens for each individual case.

Sample Stored Unit. This is the economic value of a sample aliquot that is produced and stored in the biobank. It comprises costs for both processing and storing each type of sample (DNA, plasma or PBMC). The sum of the cost of all stored samples is the inventory value of the biobank. Due to the specific characteristics of the biobank production process (in which the stored samples might not lose value or quality for years and are indistinctly delivered to researchers), we used the weighted average cost formula [6] to calculate the value of a 'sample stored unit' (see online suppl. table 1 for details; see www.karger.com/doi/10.1159/000362796 for all online suppl. material).

Sample Aliquot. This is the cost of each portion of a sample ready for delivery. It covers the value of each sample stored plus all costs involved in the sample management up to the point when it is ready for distribution, according to the specifications required by the researchers. This cost is calculated by adding the sample stored unit cost to the corresponding cost incurred at the stage of sample selection and preparation for distribution.

Order Management and Shipping. This includes all costs incurred when a sample request is received and the sample aliquots are sent out to researchers.

\section{Calculation of Direct and Indirect Costs}

The cost elements of BNADN were identified and classified into direct and indirect (overhead) costs. Direct costs include those consumptions directly and indisputably associated with production. All the consumables (e.g. tubes, plugs, labels, disposable pipettes, etc.) and reagents (e.g. isopropanol, ethanol, dimethyl sulfoxide, etc.) to be used in the biobank are included in this group of costs. Most direct costs are also variable for the biobank activity, as they vary proportionally to the volume of biospecimens handled. In turn, indirect costs include those resources consumed and needed for the general tasks required by the various types of samples obtained. Three broad groups of costs are included. The first is personnel costs, which comprise the total cost (i.e. total salary plus health and social insurance cost and taxes) of the staff directly involved in the activities of the biobank (e.g. lab manager, administrative and/or laboratory technicians). Some biobank staff combine their activity in the biobank with other hospital or university jobs, so an allocation rate was required to distribute the cost of staff among the different tasks performed (biobank activities and others) and among the different stages of the biobank production process. The second is facilities and equipment: the former comprises the costs of depreciation, rental and maintenance related to the use of spaces (office, laboratory and storage rooms), and the latter includes the costs for depreciation, rental and maintenance of equipment, furniture and all the assets used in biobanking. Finally, there are other costs which basically involve expenditures for telephone, cleaning services, electricity, nitrogen consumption, office supplies and disposal of biological waste. Of note, most indirect costs are almost always fixed costs for the biobank activity (i.e. costs that do not vary significantly as do the number of samples handled within a relevant range of volume).

As soon as all cost elements that participate in calculations are classified, each and every one of them must be allocated to each stage of the production process based on its real consumption at each stage (tables 1,2$)$. 
Table 1. Allocation and distribution of costs to biobank inventories for the year 2011 (in EUR) at BNADN

\begin{tabular}{|c|c|c|c|c|c|c|c|}
\hline Cost elements & $\begin{array}{l}\text { 1st stage: } \\
\text { collection } \\
\text { cases }\end{array}$ & DNA & plasma & PBMC & DNA & plasma & PBMC \\
\hline \multicolumn{8}{|l|}{ Direct costs } \\
\hline Blood collection tubes & $1,698.24$ & - & - & - & \multirow{9}{*}{$\begin{array}{l}\text { no } \\
\text { direct } \\
\text { costs }\end{array}$} & \multirow{9}{*}{$\begin{array}{l}\text { no } \\
\text { direct } \\
\text { costs }\end{array}$} & \multirow{9}{*}{$\begin{array}{l}\text { no } \\
\text { direct } \\
\text { costs }\end{array}$} \\
\hline Sample transportation & $12,736.80$ & - & - & - & & & \\
\hline Cell separation & - & - & $6,034.72$ & $12,144.54$ & & & \\
\hline Cell preservation & - & - & - & $3,060.23$ & & & \\
\hline Identification and traceability at room temperature & 326.62 & 108.87 & 108.87 & 108.87 & & & \\
\hline Identification and traceability of frozen products & - & - & - & $1,779.26$ & & & \\
\hline 2D-coded microtubes & - & $9,052.08$ & $6,034.72$ & - & & & \\
\hline Conductive-filtered tips for aliquoting & - & 858.89 & 858.89 & - & & & \\
\hline FTA paper & - & $14,570.90$ & - & - & & & \\
\hline Total direct costs & $14,761.66$ & $36,395.30$ & $7,586.91$ & $17,895.55$ & & & \\
\hline
\end{tabular}

\section{Indirect costs}

Equipment (depreciation and maintenance)

Automated DNA extractor

Spectrophotometer

Liquid nitrogen containers

Secured shelves for room temp. storage

\begin{tabular}{|c|c|c|c|c|c|c|}
\hline Subtotal 1. - indirect costs & - & $45,200.00$ & - & 300.00 & - & $17,600.00$ \\
\hline Automated liquid handler & - & $19,440.00$ & - & & - & - \\
\hline 2D microtube tracker system & - & $1,800.00$ & - & & - & - \\
\hline Subtotal 2. - indirect costs & - & $21,240.00$ & - & 13 , & 284.80 & - \\
\hline \multicolumn{7}{|l|}{ Personnel } \\
\hline Technicians & $5,237.47$ & $36,662.30$ & & & $2,618.73$ & \\
\hline \multicolumn{7}{|l|}{ Facilities } \\
\hline Space used & 945.00 & $12,150.50$ & & & $6,750.00$ & \\
\hline \multicolumn{7}{|l|}{ Equipment (depreciation and maintenance) } \\
\hline Sample management software (LIMS) & 280.00 & $1,120.00$ & & & 280.00 & \\
\hline Personal computers & 200.00 & 300.00 & & & - & \\
\hline Electricity & 260.00 & $1,800.00$ & & & 360.00 & \\
\hline Office supplies & $1,109.00$ & - & & & - & \\
\hline Disposal of biological waste & - & $1,420.44$ & & & - & \\
\hline Subtotal 3. - indirect costs & $16,351.12$ & $76,528.14$ & & & $10,229.53$ & \\
\hline Total indirect costs & $16,351.12$ & $142,968.14$ & & & $41,414.33$ & \\
\hline Total costs (direct + indirect $)$ & $31,212.78$ & $204,845.90$ & & & $41,414.33$ & \\
\hline
\end{tabular}


Table 2. Allocation and distribution of costs to sample requests for the year 2011 (in EUR) for BNADN

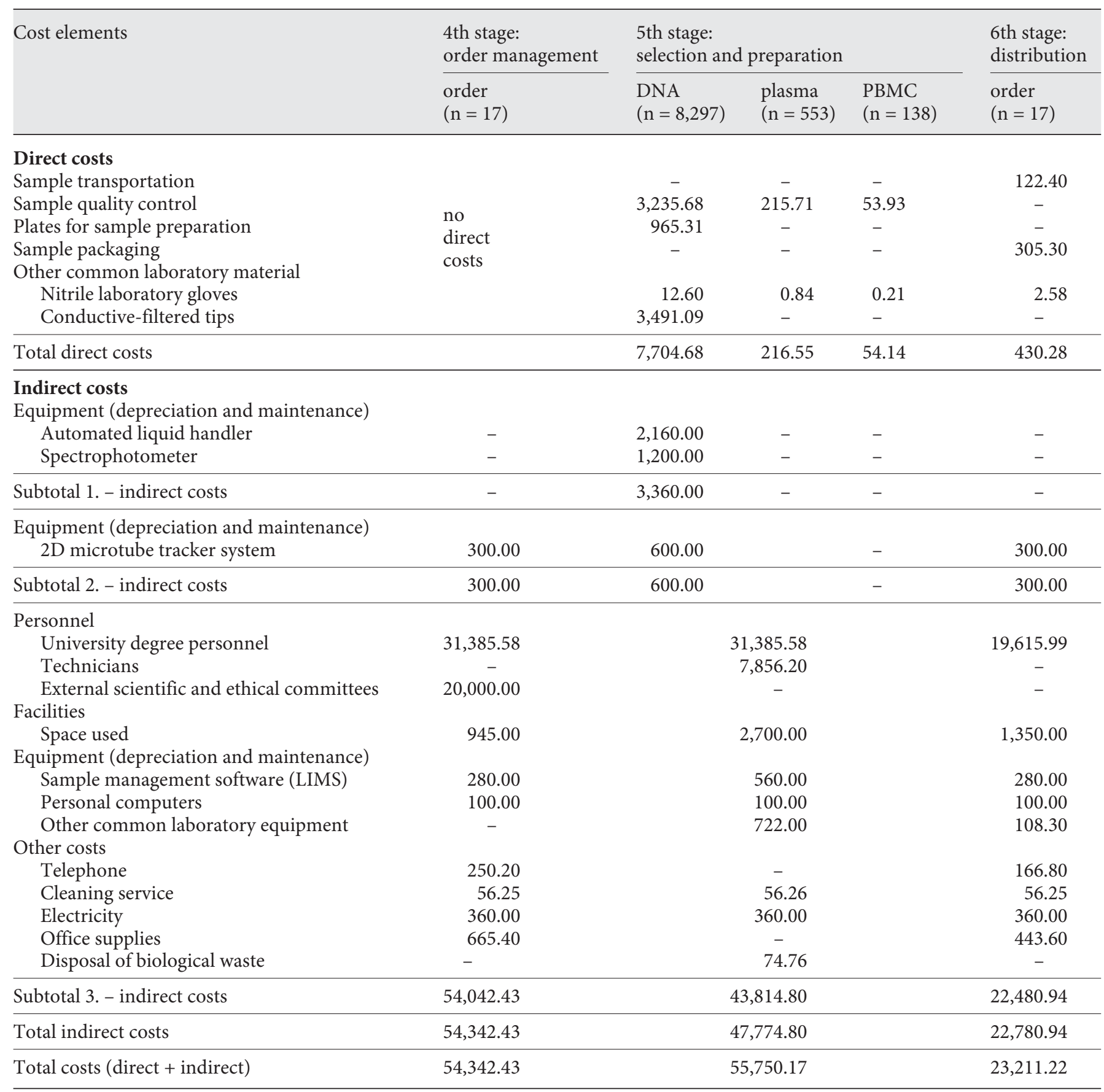

As a final step, the overall cost of some of the stages has to be distributed and assigned to the three individual products obtained. Within those stages termed 'collection of cases' and 'processing of biospecimens' the distribution takes into account the usage of labour, i.e. it considers the labour hours required for each of the products (the average normal labour dedication is $50 \%$ for DNA, $20 \%$ for plasma and $30 \%$ for PBMC). In the third stage, 'storage of sample products', cost distribution is not required. The total cost of 'sample selection and preparation for delivery' stage is distributed proportionally to the weight of each sample product within a case (e.g. the average normal sample production in a case is: 120 sample aliquots for DNA, 8 sample aliquots for plasma and 2 sample aliquots for PBMC). Finally, the distribution of the cost of the stages order management and distribution are not required. 
Table 3. Costs associated with case processing for the year 2011 (in EUR) at BNADN

\begin{tabular}{lllll}
\hline & $\begin{array}{l}\text { Total costs: collection } \\
+ \text { processing of } \\
\text { biospecimens }\end{array}$ & $\begin{array}{l}\text { Units } \\
\text { processed, } \\
\mathrm{n}\end{array}$ & $\begin{array}{l}\text { Unitary } \\
\text { cost }\end{array}$ & $\begin{array}{l}\text { Cost per } \\
\text { product }\end{array}$ \\
\hline DNA & $150,637.19$ & 212,280 & 0.71 & 85.15 \\
Plasma & $35,203.66$ & 14,152 & 2.49 & 19.91 \\
PBMC & $50,217.83$ & 3,538 & 14.19 & 28.38 \\
\hline
\end{tabular}

Costs of case

$\begin{array}{llll}\text { processing } \quad 236,058.68 & 1,769 & 133.44\end{array}$

\section{Results}

\section{Cost of Individual Cost Objects}

Cost of Case Processing

As mentioned above, the calculation of case processing costs includes the costs incurred at the collection of the case, as well as the costs incurred at the processing of the biospecimens. Both are required to obtain the three different products, e.g. DNA, plasma and PBMC.

Accordingly, the unitary cost of 'case processing' for each sample product (EUR 0.71 for $1.5 \mu \mathrm{g}$ of DNA, EUR 2.49 for $500 \mu \mathrm{l}$ of plasma and EUR 14.19 for PBMC cryovial) was calculated by dividing the total cost for each product by the number of sample units obtained from them (table 3). Likewise, the unitary cost of case processing (EUR 133.44 per case collected) was determined by dividing the total cost of case processing by the total number of cases processed (table 3 ).

Cost of Inventory Maintenance per Year. The cost object 'inventory maintenance per year' only includes indirect costs corresponding to the storage of sample products. Thus, the cost incurred for maintaining the full collection of samples stored was EUR 41,414.33 for the year 2011 (table 1).

Cost of a Sample Stored Unit. The value of the inventory was measured using historical production costs because the value increases each year by the amount of the production cost ('case processing') plus the cost of storing the processed samples ('inventory maintenance per year') within that same year, and it decreases with the costs assigned to the samples delivered. Online supplementary tables 1-3 describe how calculations for inventory valuation from 2005 to 2011 were carried out for DNA, plasma and PBMC sample aliquots, respectively. Similarly, the number of sample stored units (i.e. aliquots) within the inventory in 2011 was calculated by

Implementation of a Cost-Accounting

Model in a Biobank adding to the number of samples within the inventory at the end of 2010 (December 31, 2010), the number of samples processed in 2011, minus the samples delivered in 2011 (table 4). Therefore, the cost for each stored DNA sample at the end of 2011 (EUR 0.71) was calculated by dividing the value of the DNA collection inventory at the end of 2010 (EUR 1,916,483.32) plus the total cost of case processing and storage during 2011 (EUR $165,541.10)$ by the number of DNA samples in the inventory at the end of $2010(2,732,959)$ plus the number of samples processed during 2011 (212,280; table 4). In a similar way, the value of those DNA sample aliquots that were delivered in 2011 (EUR 5,864.98) was calculated by multiplying the number of DNA samples delivered in $2011(8,297)$ by the cost of a DNA 'sample stored unit' in 2011 (EUR 0.71; table 4). Finally, the value of the DNA inventory at the end of 2011 (EUR 2,076,159.44) was obtained by adding the value of the DNA collection inventory at the end of 2010 (EUR 1,916,483.32) plus the total cost of case processing and storage during 2011 (EUR 165,541.10), minus the total cost of the DNA units that were delivered in 2011 (EUR 5,864.98). Details about the value of the 'sample stored unit' and the inventory value of plasma and PBMC obtained for the years 2010 and 2011 are also specified in table 4 . These calculations were extended from the years 2005 to 2011 and are fully described in online supplementary tables $1-3$.

Cost of a Sample Aliquot. To calculate costs associated with a sample aliquot being distributed, costs from the moment an order is received until sample delivery, including all the cost elements consumed by the biobank when managing an order, were allocated and distributed within three different stages: 'order management', 'sample aliquot selection and preparation' and 'distribution of orders' (table 2). Therefore, the cost object 'sample aliquot' includes the unitary cost corresponding to selecting and preparing a sample aliquot for distribution within the year, plus the previously calculated cost of a 'sample stored unit' (table 5). The former is calculated by dividing the total cost of selecting a sample and preparing it for distribution by the number of samples delivered in the current year. Therefore, the cost of each DNA sample aliquot in 2011 (EUR 6.99) was obtained by adding the DNA unitary cost (EUR 6.28) for selecting and preparing samples for distribution to the DNA 'sample stored unit' cost (EUR 0.71).

Cost of Order Management and Shipping. Table 2 provides a detailed description of the total costs associated with order management and distribution of the sample 
Table 4. Costs of stored sample units during the year 2011 at BNADN

\begin{tabular}{|c|c|c|c|c|c|c|}
\hline & \multicolumn{2}{|l|}{ DNA } & \multicolumn{2}{|l|}{ Plasma } & \multicolumn{2}{|l|}{ PBMC } \\
\hline & End of 2010 & End of 2011 & End of 2010 & End of 2011 & End of 2010 & End of 2011 \\
\hline \multicolumn{7}{|l|}{ Total costs of case processing and } \\
\hline & $442,636.86$ & $165,541.10$ & $99,987.43$ & $35,203.66$ & $142,631.53$ & $50,217.83$ \\
\hline Sample stored unitary costs, EUR & 0.70 & 0.71 & 2.53 & 2.56 & 16.04 & 16.33 \\
\hline Value, EUR & $16,057.08$ & $5,864.98$ & $3,865.67$ & $1,415.32$ & $6,121.41$ & $2,257.80$ \\
\hline \multicolumn{7}{|l|}{ Inventory } \\
\hline Total value, EUR & $1,916,483.32$ & $2,076,159.44$ & $461,384.85$ & $501,014.75$ & $730,617.59$ & $799,246.47$ \\
\hline Samples, n & $2,732,959$ & $2,936,943$ & 182,197 & 195,796 & 45,549 & 48,949 \\
\hline
\end{tabular}

aliquots ready for delivery. This cost is directly dependent on the number of orders received by the biobank, but it is independent from the number of samples requested. Therefore, calculating the 'order management and shipping' unitary cost for the year 2011 is as simple as adding the cost of 'order management' (EUR 54,342.43) to the cost of 'distribution' (EUR 23,211.22; table 2) and dividing the resulting amount by the number of orders received and processed during the year (17 in 2011). Thus, the unitary cost for the year 2011 was EUR 4,561.98 per order. Of note, the 'sample aliquot' unitary cost (table 5) and the unitary cost per order must be taken into account to calculate the cost incurred for a specific order. The total cost of an order is calculated by multiplying the number of sample aliquots requested by the cost of each sample aliquot, and adding to the resulting amount the cost of order management and shipping. For example, the total cost of an order of 500 DNA sample aliquots for a specific research project is associated with a final value (EUR $8,055.98)$ that represents the total cost of the 'sample aliquots' for this order $(500 \times$ EUR $6.99=$ EUR 3,494.00 $)$ plus the cost of 'order management and shipping' (EUR 4,561.98).

\section{Cost Structure Analysis}

In the previous section we identified the operative activities that drive the total cost of the biobank and the variable versus fixed nature of each cost. The results obtained highlighted the convenience to examine the cost behaviour for different scenarios of production volume, in order to predict cost variations given a one-unit change in the drivers of that cost. This may be helpful to analyse, for example, how cost would be affected by the decision
Table 5. Costs of sample aliquots prepared for distribution during the year 2011 (in EUR) at the BNADN

\begin{tabular}{lrrrl}
\hline & DNA & Plasma & PBMC & Total \\
& & & & \\
\hline Samples delivered, $\mathrm{n}$ & 8,297 & 553 & 138 & 8,988 \\
\hline $\begin{array}{l}\text { Sample selection and preparation for distribution } \\
\quad \text { Total costs }\end{array}$ & $52,071.61$ & $2,950.35$ & 728.21 & $55,750.17$ \\
$\quad$ Unitary costs & 6.28 & 5.34 & 5.28 & - \\
$\begin{array}{l}\text { Samples stored } \\
\quad \text { Unitary costs }\end{array}$ & 0.71 & 2.56 & 16.33 & - \\
$\begin{array}{l}\text { Sample aliquots } \\
\text { Unitary costs }\end{array}$ & 6.99 & 7.90 & 21.61 & - \\
\hline
\end{tabular}

to change the size of the biobank, to cut costs or to make changes in the product portfolio. In order to build different scenarios to analyse cost behaviour, two cost functions were drawn from the results of the model implemented and proposed for each product: total production cost for a specific product (collection of cases and processing of biospecimens costs) and total request cost for a specific order (reception, preparation and distribution costs). The stage of storage of sample products is omitted for this cost structure analysis since its costs are directly attached and they increase the value of the inventory independently of the volume of the production activity. Following, the total production cost function for a specific product is:

$$
T P C_{k}=I C P_{k}+\left(\sum_{a}^{z} I C P \times \frac{L H_{k}}{\sum_{a}^{z} L H}\right)+\left(D C P_{k} \times S P_{k}\right) .
$$


Therefore, the total production cost for a specific product $\left(\mathrm{TPC}_{\mathrm{k}}\right)$ is driven, firstly, by the indirect costs of collecting cases and processing specific biospecimens for each type of product $\left(\mathrm{ICP}_{\mathrm{k}}\right)$, and these costs are fixed, i.e. they are independent of the number of samples processed. Secondly, it is driven by the indirect cost of collecting cases and processing biospecimens shared among all the different types of products generated in the biobank ( $(\mathrm{ICP})$; this indirect cost is distributed among each production in proportion to the labour hours required for the product $\left(\mathrm{LH}_{\mathrm{k}}\right)$ within the total number of labour hours $(\Sigma \mathrm{LH})$ for the period. Finally, total production costs are driven by the direct costs of collecting cases and processing biospecimens for a specific product $\left(\mathrm{DCP}_{\mathrm{k}}\right)$; such costs vary according to the number of samples of the product produced within the period $\left(\mathrm{SP}_{\mathrm{k}}\right)$. The value of $\mathrm{DCP}_{\mathrm{k}}$ comes from the specific direct costs of the stage of processing that biospecimen plus the proportion of the direct costs of case collecting specifically assigned to that biospecimen. Taking into account the data provided for the biobank in table 1, the total production cost function for DNA $\left(T P C_{d n a}\right)$ is the following:

$$
\begin{aligned}
T P C_{d n a} & =\text { EUR 45,200.00 } \\
& +\left(\text { EUR 92,879.26 } \times \frac{L H_{d n a}}{L H_{d n a}+L H_{p l}+L H_{p b m c}}\right) \\
& +\left(\text { EUR2 } 1,240.00 \times \frac{L H_{d n a}}{L H_{d n a}+L H_{p l}}\right)+\left(\text { EUR } 0.21 \times S P_{d n a}\right) .
\end{aligned}
$$

The $\mathrm{ICP}_{\mathrm{dna}}$ implies that regardless of the products and samples obtained, the biobank will incur fixed costs of EUR 45,200.00 in DNA production (the cost coming from the depreciation and maintenance of the automated DNA extractor and the spectrophotometer). Furthermore, the total production cost for DNA will also depend on the labour hours employed for plasma $\left(\mathrm{LH}_{\mathrm{pl}}\right)$ and PBMC $\left(\mathrm{LH}_{\mathrm{pbmc}}\right)$ because they share a cost of EUR $92,879.26$ (among other costs, it includes personnel, space usage, LIMS, cleaning service and electricity costs). It will additionally depend on the labour hours of plasma because they share a cost of EUR 21,240.00 (automated liquid handler and 2D microtube tracker system). These costs are distributed to each product proportionally to the number of labour hours involved in the production of each product from the total hours consumed. Finally, for every DNA sample produced, the biobank will expend EUR 0.21. Similarly, the total production cost functions for plasma $\left(\mathrm{TPC}_{\mathrm{pl}}\right)$ and PBMC $\left(\mathrm{TPC}_{\mathrm{pbmc}}\right)$ would be as follows:

Implementation of a Cost-Accounting Model in a Biobank

$$
\begin{aligned}
& T P C_{p l}=\left(\text { EUR92,879.26 } \times \frac{L H_{p l}}{L H_{d n a}+L H_{p l}+L H_{p b m c}}\right) \\
& +\left(\operatorname{EUR} 21,240.00 \times \frac{L H_{p l}}{L H_{d n a}+L H_{p l}}\right)+\left(\text { EUR } 0.74 \times S P_{p l}\right) \\
& T P C_{p b m c}=\left(\text { EUR 92,879.26 } \times \frac{L H_{p b m c}}{L H_{d n a}+L H_{p l}+L H_{p b m c}}\right) \\
& +\left(\text { EUR 6.31 } \times S P_{p b m c}\right) \text {. }
\end{aligned}
$$

Of note, $\mathrm{TPC}_{\mathrm{pl}}$ and $\mathrm{TPC}_{\mathrm{pbmc}}$ do not consume resources that can be considered fixed production costs specific to plasma $\left(\mathrm{ICP}_{\mathrm{pl}}\right)$ and $\mathrm{PBMC}\left(\mathrm{ICP}_{\mathrm{pbmc}}\right)$, respectively. This means that if the biobank decides to stop the production of plasma and/or PBMC, some of their production costs will disappear (DCP) and some will be charged to the other products being produced $(\Sigma \mathrm{ICP})$. As mentioned above in $\mathrm{TPC}_{\mathrm{dna}}$, there are also some resources that are shared only between plasma and DNA (the penultimate of $\mathrm{TPC}_{\mathrm{pl}}$ ).

Based on previous cost functions, three different production scenarios have been simulated for 1,500, 5,000 and 7,500 cases, respectively (table 6). Through the analysis of these scenarios it can be seen how the increase in production (information is provided for different products: cases, DNA, plasma and PBMC samples) has different effects on the behaviour of the cost, depending on the type of cost that is considered. Direct costs do not vary per unit in the three different scenarios, but indirect costs clearly decrease per unit.

In turn, the total request cost for a specific order depends on the order management, sample selection and preparation for distribution, and distribution of sample aliquots, and it can be calculated as per the following formula:

$$
\begin{aligned}
\text { TORC }_{k i}= & D D+\left(\frac{I M D}{\sum_{1}^{n} O r}\right)+\left(I P_{k} \times \frac{S D_{i}}{\sum_{1}^{n} S D_{k}}\right) \\
& +\left(\sum_{a}^{z} I P \times \frac{S D_{i}}{\sum_{1}^{n} S D}\right)+\left(D P_{k} \times S D_{i}\right) .
\end{aligned}
$$

Therefore, the total request cost for a specific order of a specific product $\left(\mathrm{TORC}_{\mathrm{ki}}\right)$ is driven, firstly, by the direct costs of the distribution stage (DD). These costs are fixed for each order and do not vary depending on the type of product requested, the number of samples included in that order, or the number of orders received and distributed within the period. Secondly, $\mathrm{TORC}_{\mathrm{ki}}$ is driven by the indirect costs of the stage of the 'order management' and the stage of 'distribution' (IMD) which are distributed to each 
Table 6. Total production costs for a specific product $\left(\mathrm{TPC}_{\mathrm{k}}\right)$ in three different (theoretical) scenarios

\begin{tabular}{|c|c|c|c|}
\hline & Scenario 1 & Scenario 2 & Scenario 3 \\
\hline \multicolumn{4}{|l|}{ Case } \\
\hline Cases, $\mathrm{n}$ & 1,500 & 5,000 & 7,500 \\
\hline \multicolumn{4}{|l|}{ Costs of case processing per unit, EUR } \\
\hline $\mathrm{ICP}_{\text {case }}$ & 30.13 & 9.04 & 6.03 \\
\hline$\sum \mathrm{ICP}_{\text {case }}$ & 76.08 & 22.82 & 15.22 \\
\hline $\mathrm{DCP}_{\text {case }}$ & 43.32 & 43.32 & 43.32 \\
\hline Total production costs per case, EUR & 149.54 & 75.19 & 64.57 \\
\hline \multicolumn{4}{|l|}{$D N A$} \\
\hline DNA sample units, $\mathrm{n}$ & 180,000 & 600,000 & 900,000 \\
\hline \multicolumn{4}{|l|}{ Production costs components for a DNA sample, EUR } \\
\hline $\mathrm{ICP}_{\mathrm{dna}}$ & 0.25 & 0.08 & 0.05 \\
\hline$\sum \mathrm{ICP}_{\text {shared }}$ & 0.34 & 0.10 & 0.07 \\
\hline $\mathrm{DCP}_{\text {dna }}$ & 0.21 & 0.21 & 0.21 \\
\hline Total production costs per DNA sample, EUR & 0.80 & 0.38 & 0.32 \\
\hline \multicolumn{4}{|l|}{ Plasma } \\
\hline Plasma sample units, $\mathrm{n}$ & 12,000 & 40,000 & 60,000 \\
\hline \multicolumn{4}{|c|}{ Production costs components for a plasma sample, EUR } \\
\hline $\mathrm{ICP}_{\mathrm{pl}}$ & - & - & - \\
\hline$\sum \mathrm{ICP}_{\text {shared }}$ & 2.05 & 0.62 & 0.41 \\
\hline $\mathrm{DCP}_{\mathrm{pl}}$ & 0.74 & 0.74 & 0.74 \\
\hline Total production costs per plasma sample, EUR & 2.80 & 1.36 & 1.16 \\
\hline \multicolumn{4}{|l|}{$P B M C$} \\
\hline PBMC sample units, $\mathrm{n}$ & 3,000 & 10,000 & 15,000 \\
\hline \multicolumn{4}{|c|}{ Production costs components for a PBMC sample, EUR } \\
\hline $\mathrm{ICP}_{\mathrm{pbmc}}$ & - & - & - \\
\hline$\sum \mathrm{ICP}_{\text {shared }}$ & 9.29 & 2.79 & 1.86 \\
\hline $\mathrm{DCP}_{\mathrm{pbmc}}$ & 6.31 & 6.31 & 6.31 \\
\hline Total production costs per PBMC sample, EUR & 15.60 & 9.10 & 8.17 \\
\hline
\end{tabular}

order proportionally to the total number of orders received and distributed during the period ( $\Sigma \mathrm{Or}$ ). Thirdly, the total request costs is also driven by the indirect costs associated to sample selection and preparation that are product-specific $\left(\mathrm{IP}_{\mathrm{k}}\right)$ and proportional to the number of samples prepared for that order $\left(\mathrm{SD}_{\mathrm{i}}\right)$ and the total samples of that product prepared within the period $\left(\Sigma S_{\mathrm{k}}\right)$. Fourthly, the TORC $_{\mathrm{ki}}$ cost is also dependent on the indirect cost of the stage 'sample selection and preparation' that is shared with other orders ( $(\mathrm{IP})$ that are also charged proportionally to the number of samples of the specific order $\left(\mathrm{SD}_{\mathrm{i}}\right)$ and the total number of samples prepared and distributed $(\Sigma \mathrm{SD})$ within the period. Finally, the $\mathrm{TORC}_{\mathrm{ki}}$ is also driven by the direct costs specifically associated to 'sample selection and preparation' for that product $\left(\mathrm{DP}_{\mathrm{k}}\right)$ and the number of samples contained in the specific order $\left(\mathrm{SD}_{\mathrm{i}}\right)$.

Once the BNADN biobank data presented in table 2 is taken into account, the total request cost for a specific order of DNA $\left(\right.$ TORC $\left._{\text {dna-i }}\right)$ would be as follows:

$$
\begin{aligned}
\text { TORC }_{\text {dna-i }} & =\text { EUR 25.31 }+\left(\frac{\text { EUR } 54,342.43+\text { EUR 22,780.94 }}{\sum_{1}^{n} \text { Or }}\right) \\
& +\left(\text { EUR } 3,360.00 \times \frac{S D_{i}}{\sum_{1}^{n} S D_{d n a}}\right) \\
& +\left(\text { EUR } 43,814.80 \times \frac{S D_{i}}{\sum_{1}^{n} S D}\right) \\
& +\left(\text { EUR } 600.00 \times \frac{S D_{i}}{\sum_{1}^{n} S D_{\text {dna }}+\sum_{1}^{n} S D_{p l}}\right) \\
& +\left(\text { EUR } 0.93 \times S D_{i}\right) .
\end{aligned}
$$

In the model proposed, DD costs (EUR 25.31) are derived from sample transportation, sample quality control and other common laboratory materials. IMD costs (EUR $54,342.43+22,780.94)$ consist of the whole indirect cost of the stages of 'order management and distribution of samples'. $\mathrm{IP}_{\mathrm{dna}}$ costs (EUR 3,360.00) come from the deprecia- 
Table 7. Total sample request costs for a specific order $\left(\mathrm{TORC}_{\mathrm{ki}}\right)$ in three different (theoretical) scenarios

Scenario 1 Scenario 2 Scenario 3

\begin{tabular}{lcll}
\hline $\begin{array}{l}\text { Total number of } \\
\text { orders, } \mathrm{n}\end{array}$ & 15 & 30 & 45 \\
\hline $\begin{array}{l}\text { Total sample request costs, EUR } \\
\text { DNA }\end{array}$ & $8,836.30$ & $4,663.38$ & $3,272.41$ \\
Plasma & $8,323.63$ & $4,272.04$ & $2,921.52$ \\
PBMC & $8,283.00$ & $4,251.73$ & $2,907.97$ \\
\hline
\end{tabular}

tion and maintenance of the automated liquid handler and the spectrophotometer. $\mathrm{IP}_{\mathrm{dna}} \mathrm{pl}$, pbmc costs (EUR 43,814.80) derive from those indirect costs of the stage of 'sample preparation for distribution' which are shared by DNA, plasma and PBMC products. IP $_{\text {dna, pl }}$ costs (EUR 600.00) come from the indirect costs that are shared by the DNA and plasma products prepared within the period. Finally, $\mathrm{DP}_{\mathrm{dna}}$ costs (EUR 0.93) derive from the direct costs associated with preparation of the DNA sample aliquots for distribution (sample quality control, plates for sample preparation and other common laboratory material).

Similarly, the total request cost for a specific order of plasma $\left(\mathrm{TORC}_{\mathrm{pl-}-\mathrm{i}}\right)$ and PBMC $\left(\mathrm{TORC}_{\mathrm{pbmc-i}}\right)$ would be calculated as follows:

$$
\begin{aligned}
& \operatorname{TORC}_{p l-i}=\mathrm{EUR} 25.46+\left(\frac{\mathrm{EUR} 54,342.43+\mathrm{EUR} 22,780.94}{\sum_{1}^{n} \mathrm{Or}}\right) \\
& +\left(\operatorname{EUR} 43,814.80 \times \frac{S D_{i}}{\sum_{1}^{n} S D}\right) \\
& +\left(\operatorname{EUR} 600.00 \times \frac{S D_{i}}{\sum_{1}^{n} S D_{d n a}+\sum_{1}^{n} S D_{p l}}\right) \\
& +\left(\text { EUR } 0.39 \times S D_{i}\right) \\
& \text { TORC }_{p b m c-i}=\operatorname{EUR25.46}+\left(\frac{\text { EUR 54,342.43+EUR22,780.94 }}{\sum_{1}^{n} \text { Or }}\right) \\
& +\left(\operatorname{EUR} 43,814.80 \times \frac{S D_{i}}{\sum_{1}^{n} S D}\right)+\left(\text { EUR } 0.39 \times S D_{i}\right) .
\end{aligned}
$$

Based on the above costs functions, three different request scenarios have been simulated for 15, 30 and 45 orders, respectively (table 7). The analysis of such scenarios shows that an increase in the number of orders delivered has different effects on the behaviour of the cost. Direct unitary costs (distribution and sample preparation) remain constant for the three different scenarios, but indirect costs per order decrease as the number of orders increases. The same type of analysis could be carried out for each of the sample aliquots requested.

\section{Discussion}

Although biobanks around the world frequently are non-profit organizations, they still must consider economic balance if they want to manage their activities optimally. Whenever expenses are greater than revenues (the budget), biobank sustainability will be difficult in the medium/ long term. The results obtained in this study can trigger discussion on some key implications concerning both the biobank managers and the research and development funding agencies that support biobank sustainability.

Overall biobanking activities involve high resourceconsuming activities with a significant proportion of fixed costs $(79 \%$ in the case of BNADN presented here for 2011). For more detailed analysis of these figures, data associated with production and request activities must be separately analysed.

Regarding production activities (storage not included), the costs functions showed that DNA production is the process with the highest fixed (69\%) and the lowest variable $(31 \%)$ production costs. This fits the general principle that the higher the total fixed cost, the lower the variable unitary cost. In turn, storage is a central activity where $100 \%$ of the costs are fixed, even though this cost only represents $10 \%$ of the total resources consumed in one year. A more detailed analysis of the costs associated with the production process showed that automation in the biobank represented one of the highest costs, and it is totally independent of the number of cases processed (fixed cost). For BNADN, depreciation and maintenance of the automated DNA extractor device is almost $50 \%$ of the fixed costs associated with DNA production. The size of the workforce (the greatest fixed costs of the biobank) is not always determined by management. However, management may intervene in deciding whether or not to purchase devices. Such a decision might be better taken if it is preceded by the calculation for each potential alternative (e.g. automated vs. manual production) of the different unitary production costs associated to the expected workflow (e.g. number of cases per year). In any case, caution should be taken not to compromise the quality of the samples and data (e.g. reduction in automation could place the traceability of samples at risk). 
Determining the optimal level of sample production with the available resources would help to plan the appropriate size of the biobank collections of samples (e.g. the number of cases processed, the type of samples archived in the biobank, and the number and type of different disease collections). Therefore, fluctuations in the workflow and the workload (e.g. 1,500 vs. 7,500 cases processed per year) reveal that unitary fixed production costs decrease in parallel to the increase in the amount of cases collected and processed. Obviously, working at the maximal production capacity of the biobank will always reduce unitary costs per product. Thus, if biobank activities are planned to achieve an appropriate ratio between the production capacity and the number of cases to be collected, it is crucial for the biobank to make good strategic decisions and implement an adequate working plan. Further analyses showed that some products were more dependent on fixed costs than others. Based on the data obtained, it can be argued that processing products with high fixed costs, such as DNA, would be more efficient in specialized medium- to large-sized biobanks which are able to process large numbers of cases with high-throughput automated technologies.

The high fixed costs observed for the production process raises other interesting questions regarding the optimal structure of biobanks. For instance, could concentration strategies be supported by our data, upon comparing the costs of big centralized biobanks or biobank networks, against multiple (usually oversized) small biobanks attached to individual centers? The total budget could be optimized through usage of common facilities (e.g. centralized DNA production processes or product archives) and the cooperation of multiple biobanks within regional/ national biobank networks [9] in obtaining strategic sample collections. Adequate decisions on these matters may help to address the financial sustainability of biobanks.

Regarding costs associated with request activities ('order management', 'sample aliquot selection and preparation' and 'distribution') it should be noted that such costs are strongly dependent on the number of aliquots requested since they are greatly influenced by the fixed costs. In the BNADN model presented here, the cost of the external committees is considered to be a fixed indirect cost that represents more than one third (37\%) of the cost associated with the management of the orders for samples for the year 2011. Ethical and scientific external review of the research projects that support orders is recommended [10] or even mandatory in some countries [11]. Therefore, strategic decisions to reduce costs associated to this process should be carefully taken at the level of research and development agencies (e.g. ministries of science) who could decide to, for example, promote the existence of centralized review boards. In any case, managerial decisions like deciding to pay the committees per each of the reviews performed will reduce the high variability of cost per unitary order which is now associated with the total number of orders processed each year. Moreover, decisions like outsourcing or centralizing (e.g. central facility within biobank networks) part of the final processes associated with the management of sample orders and sample aliquot distribution would also contribute to significantly reduce biobank expenses in this regard.

Despite all of the above considerations, it should be noted that distribution of samples at maximal capacity of a biobank will reduce the cost per sample aliquot distributed. Although this can hardly be planned in the short term because it is mostly dependent on the interest researchers have on the biobank inventory or even on research and development national policies (e.g. reduction of public or private budgets dedicated to research, the promotion of research on a specific type of disease, etc.). In any case, whenever biobank managers adequately plan the strategic samples to be collected on a medium-/long-term plan, they can influence the future success of the biobank inventory by adjusting the biobank offer of samples to the needs of researchers.

Apart from ethical and legal considerations about the rationale of biobanks [11, 12], another current issue of debate between researchers and biobank professionals is the prospective versus retrospective production and distribution models. In this regard, since the costs incurred by a biobank at case collection, biospecimen processing and sample storage are high, research and development funding agencies may question whether it would be more reasonable to prospectively collect specific samples for a single, yet defined, order, instead of collecting and archiving biological products based on their hypothetical usage in future research projects. Vaught et al. [7] consider that a 'general collection system' implies a retrospective collection where specimens are available for any research order, while a 'project-driven system' implies a prospective collection that consists in collecting and delivering specimens on demand, in response to specific sample requests. The characteristics of a prospective model can vary slightly among different countries and organizations. Therefore, some assumptions should be made to compare the costs associated with the two different production models. The prospective model avoids costs derived from the following stages: 'product storage', 'order management', 'sample selection and preparation for distribution' and 'distribution of sample aliquots' (except the costs for sample transportation). In addition, in the prospective model, each sample requested in an order 
requires collecting and processing of a new case. In turn, all fixed costs linked to the stages of collection of cases and processing of biospecimens would be the same in both models whenever the cases are processed in facilities with similar workflows (equal number of cases per year). In the prospective model, only some variable costs such as those associated with the volume of $\mathrm{PB}$ processed (e.g. it may only be reduced to $1 \mathrm{ml}, 1 / 10$ of the usual $\mathrm{PB}$ processed in a retrospective biobank) and the number of products obtained (e.g. DNA only, instead of DNA plus plasma and PBMC) can be saved compared to the retrospective model. Based on these assumptions, the unitary production cost for a prospective research project using 500 DNA samples in an optimal theoretical scenario which processes 7,500 cases per year (e.g. scenario 3 in table 6), would be approximately EUR 23.73 [the same fixed cost $\left(\mathrm{ICP}_{\text {case }}=\mathrm{EUR} 6.03\right.$ plus $\left.\sum \mathrm{ICP}_{\text {case }}=\mathrm{EUR} 15.22\right)$ and $1 / 10$ of the variable cost of the case assigned to DNA $-10 \% \mathrm{DCP}_{\text {case }}=10 \%(43.32-$ $5.92-12.62)=$ EUR 2.48]. Thus, the total costs for the prospective project would be EUR 11,890.31 (EUR 11,865.00 linked to samples themselves plus EUR 25.31 for sample transportation). Let us point out that in the prospective scenario each sample requested involves processing of a new case. Therefore, to calculate the cost of the same order of 500 DNA samples handled in a retrospective way in a biobank, we have to consider a similar scenario that distributes 7,500 DNA samples per year corresponding to, for example, 15 orders of 500 samples each (e.g. scenario 1 in table 7). In such a scenario, the cost of the order will be the cost of the 500 DNA sample aliquots stored in the biobank inventory, e.g. EUR 355 (EUR 0.71 for each sample stored for the BNADN inventory in 2011, as detailed in table 4), plus EUR $8,836.30$ for order management and shipping (scenario 1 in table 7); this results in EUR 9,191.30, an amount that is sig- nificantly lower than the overall costs for the prospective model. Besides all of the above, DNA sample aliquots would be immediately available to researchers in the biobanking retrospective model, while researchers would be forced to wait to receive the samples for several months (or even years) in the prospective model, depending on the prevalence of the disease, size of the collection required and the effectiveness of the collecting network, and such an environment leads to extra indirect research costs.

In summary, the results presented here regarding the implementation of a cost-accounting model in a biobank have both managerial (e.g. decisions on the number and type of samples to be processed and stored) and institutional/governmental implications (e.g. decisions about the appropriate size of biobanks or concentration strategies in biobank networks). Appropriate decisions in this regard may contribute to better address the long-term financial sustainability of biobanks; to this end, analysis of cost information is simply essential. However, such strategic decisions cannot be based only on cost numbers but also need to take into account the researchers' demands as well as the strategic importance of biobanks within national/international research and development policies to support scientific and clinical research.

\section{Acknowledgements}

The National DNA Bank Carlos III is supported by grants from the Instituto de Salud Carlos III of the Ministerio de Economia y Competitividad of Spain (grants: PT13/0001/0037 and PT13/0010/0067-FEDER). The authors would like to acknowledge the work of Ana Maria Regalado Mayordomo (from the National DNA Bank Carlos III, Salamanca, Spain) and Erea Nóvoa Blanco (from University of Vigo, Orense, Spain) in the initial phases of this paper.

\section{References}

-1 Trenchard PM, Dixon R: The clinical allocation of joint blood product costs. Manage Account Res 2003;14:165-176.

-2 Eldenburg L, Krishnan R: Management Accounting and Control in Health Care: An Economics Perspective; in Chapman CS, Hopwood AG, Shields MD (eds): Handbook of Management Accounting Research. Oxford, Elsevier Science, 2007, vol 2, pp 859-883.

3 Vaught J, Rogers J, Myers K, Lim MD, Lochart N, Moore H, Sawyer S, Furman JL, Compton C: An NCI perspective on creating sustainable biospecimen resources. J Natl Cancer 2011;42: $1-7$.

4 Vaught J, Rogers J, Carolin T, Compton C: Biobankonomics: developing a sustainable business model approach for the formation of a hu- man tissue biobank. J Natl Cancer Inst Monogr 2011;42:24-31.

5 Gonzalez-Sanchez M B, Lopez-Valeiras E, Morente M, Fernandez-Lago O: Cost model for biobanks. Biopreserv Biobank 2013;11:272277.

6 International Public Sector Accounting Standard (IPSAS) 12 'Inventories'. London, International Accounting Standards Board (IASB), 2006.

7 Vaught J, Kelly A, Hewitt R, Compton C: A review of international biobanks and networks: success factors and key benchmarks. Biopreserv Biobank 2010;7:143-150.

8 Finkler SA, Ward DM, Baker JJ: Essentials of Cost Accounting for Health Care Organizations, ed 3. Sudbury, Jones \& Bartlett, 2007.
-9 SakJ, Pawlikowski J, Goniewicz M, Witt M: Population biobanking in selected European countries and proposed model for a Polish national DNA bank. J Appl Genet 2012;53:159-165.

10 International Declaration on Human Genetic Data, Resolution 15 Adopted by the General Conference at Its 32nd Session. Paris, United Nations Educational, Scientific and Cultural Organization, 2003.

-11 Arias-Diaz J1, Martín-Arribas MC, García del Pozo J, Alonso C: Spanish regulatory approach for biobanking. Eur J Hum Genet 2013;21:708712 .

12 Cambon-Thomsen A, Rial-Sebbag E, Knoppers BM: Trends in ethical and legal frameworks for the use of human biobanks. Eur Respir J 2007;30:373-382.
Implementation of a Cost-Accounting Model in a Biobank 